\title{
Nursing Care of Labor Induction in 2 Cases of Upper GI Bleeding in Pregnancy Complicated with Decompensated Cirrhosis
}

\author{
Yu Yang*, Xiaoyan Chen*, Xuyao Li”, Qiuying Lin, Pingping Quan, Jufang Lin \\ Department of Obstetrics, The Third Affiliated Hospital of Sun Yat-sen University, Guangzhou, China \\ Email: "632963792@qq.com
}

How to cite this paper: Yang, Y., Chen, X.Y., Li, X.Y., Lin, Q.Y., Quan, P.P. and Lin, J.F. (2020) Nursing Care of Labor Induction in 2 Cases of Upper GI Bleeding in Pregnancy Complicated with Decompensated Cirrhosis. Open Journal of Obstetrics and Gynecology, 10, 1741-1748. https://doi.org/10.4236/ojog.2020.10120157

Received: November 29, 2020

Accepted: December 22, 2020

Published: December 25, 2020

Copyright $\odot 2020$ by author(s) and Scientific Research Publishing Inc. This work is licensed under the Creative Commons Attribution International License (CC BY 4.0).

http://creativecommons.org/licenses/by/4.0/

\begin{abstract}
Objective: To summarize the nursing experience of induced labor of upper gastrointestinal bleeding in pregnancy complicated with decompensated cirrhosis. Methods: To treat two pregnant women with upper gastrointestinal bleeding complicated with decompensated cirrhosis with early labor induction, strengthen the nursing of upper gastrointestinal bleeding before operation, monitor changes of the patient's condition closely after operation, and strengthen the prevention and treatment of postpartum complications and health guidance. Results: No serious complications occurred in the 2 patients after operation. They were hospitalized for 11 and 17 days after operation and were discharged stably. Conclusion: The pregnant women with upper gastrointestinal bleeding complicated with decompensated cirrhosis should terminate pregnancy in time and strengthen perinatal nursing, which can improve the liver function and blood coagulation and improve the prognosis of the patients.
\end{abstract}

\section{Keywords}

Decompensated Cirrhosis, Upper GI Bleeding, Induced Labor, Nursing

\section{Introduction}

Decompensated cirrhosis refers to the development of liver cirrhosis to a certain extent, beyond the compensatory capacity of liver function. The main clinical manifestations are decreased liver function and portal hypertension caused by esophageal-gastric varices, which further lead to upper GI bleeding. When the decompensated stage of liver cirrhosis is complicated with pregnancy, the de-

${ }^{\star}$ These authors contributed equally to this work. 
compensated period is easy to be complicated with hypoproteinemia, upper GI bleeding, lack of coagulation factors, etc., so it has adverse effects on pregnancy and fetus [1]. If the upper GI bleeding in the decompensated stage of pregnancy complicated with liver cirrhosis is not treated in time, it can lead to maternal liver and kidney failure, shock, DIC and even death, which is a direct threat to the safety of pregnant women. At present, the relevant studies are mainly retrospective clinical analysis [2], case reports and so on. But there is still a lack of nursing experience. In June 2019 and October 2020, 2 patients with decompensated upper GI bleeding in early and middle pregnancy complicated with decompensated liver cirrhosis were treated in our hospital. Labor induction was offered because of the aggravation of liver cirrhosis. The nursing was summarized as follows.

\section{Clinical Data}

Patient 1: 34y, G3P1, $15^{+5}$ weeks of pregnancy, abdominal distension, poor anorexia, relieving black stool for more than 1 week, found that the fetus-free heart beat for half a day. 2 years history of hepatitis B cirrhosis. Considering diagnosis: Single stillborn fetus at $15^{+5}$ weeks of pregnancy in G3P1; Severe chronic hepatitis B; Decompensated cirrhosis; upper GI bleeding. Admission date: June 14, 2019. Physical examination on admission, $\mathrm{T} 36.9^{\circ} \mathrm{C}, \mathrm{BP} 136 / 82 \mathrm{mmHg}, \mathrm{P} 92 \mathrm{bpm}$; Binocular mild yellow staining, irregular uterine contractions. Blood test after admission showed: Leukocytosis, hemoglobin decreased, thrombocytopenia, high ALT, high TBIL, high IBIL, low ALB, high LDH, PT15.2s, APTT15.1s, high TBA; Positive stool occult blood test. Symptomatic treatments were given. Endoscopic gastric fundus vein ligation was performed under intravenous anesthesia on the second day of admission. At 17:30 on the same day, Foley catheter was placed to promote cervical ripening and induced labor. The uterine contraction was regular at 0:45 the next day, and the Foley catheter came out on its own. The stillbirth was delivered at 3:55. Intrapartum bleeding $350 \mathrm{~mL}$. BP 121 - 150/62 $93 \mathrm{mmHg}$, Intramuscular injection of carprost aminobutanol $250 \mu \mathrm{g}$, hemorrhage $24 \mathrm{~h}$ postpartum total $410 \mathrm{~mL}$. The $1^{\text {st }}$ day after delivery: Transfusion of homotypic concentrated red blood cells $2 \mathrm{U}$. Abdominal puncture and drainage were given on the $3^{\text {rd }}$ day after delivery, drainage $400 \mathrm{ml}$, and then decreased gradually, and $0 \mathrm{~m}$ Lon the $6^{\text {th }}$ day after delivery, withdrawing the drainage tube. Blood test after $9^{\text {th }}$ day after delivery returned to normal. Patient was discharged from hospital in stable condition.

Patient 2: 24y, G1P0, $11^{+4}$ weeks of pregnancy, yellow urine, poor appetite for more than one month, aggravation with edema of both lower extremities for 2 weeks, sudden hematemesis for 1 day, immune cirrhosis 8 years. Color ultrasound hints: Liver volume shrinks, a small amount of ascites. Considering diagnosis: $11^{+4}$ weeks of pregnancy, G1P0; Decompensated cirrhosis; upper GI bleeding. Admission date: Oct. 3, 2020. Physical examination on admission, $\mathrm{T} 37.3^{\circ} \mathrm{C}, \mathrm{BP} 100 / 56 \mathrm{mmHg}, \mathrm{P} 104 \mathrm{bpm}$, moderately yellow in complexion, and 
both lower limbs swollen (++). Blood test after admission showed: Leukocytosis, thrombocytopenia, high ALT, high TBIL, high D-BIL, high IBIL, low ALB, high LDH, PT16.4s, APTT14.5s, low PTA; Positive stool occult blood test. Termination of pregnancy was suggested due to severe condition, but the patient refused. Symptomatic treatments were given. After admission, the patient still had a small amount of bleeding every day and was offered a bland diet. On the $3^{\text {rd }}$ day, the amount of bleeding suddenly increased to $600 \mathrm{ml}$. Reexamination showed: hemoglobin decreased, thrombocytopenia, high ALT, high TBIL, high D-BIL, high IBIL, PT20.1s, APTT18.5s, PTA40\%, which suggested that anemia was further aggravated, and liver function and blood coagulation function were worsening. Considering the aggravation of the condition, pregnant women preferring to continued gestation would have the potentials of liver failure and DIC. Under patient's informed consents on the $3^{\text {rd }}$ day of admission, endoscopic esophagogastric variceal ligation and ultrasound-guided uterine curettage was performed under intravenous inhalation combined anesthesia. The intraoperative bleeding was $600 \mathrm{~mL}$, and the uterine cavity packing balloon was placed to stop the bleeding. $4 \mathrm{U}$ of concentrated red blood cells of the same type was infused and fresh frozen plasma $400 \mathrm{~mL}$ was used. Transfer to ICU monitoring after operation. Postoperative vital signs were stable. The uterine packing balloon was removed at $24 \mathrm{~h}$ after operation, and postpartum hemorrhage $(650 \mathrm{~mL})$ was performed at $24 \mathrm{~h}$. The $3^{\text {rd }}$ day after operation, the biochemical indexes tended to be stable. The patient were pre-transferred to ICU and discharged on the $14^{\text {th }}$ day after operation.

\section{Nursing}

\subsection{Nursing of Upper GI Bleeding}

1) Vital signs monitoring: The condition of the patients with decompensation of liver cirrhosis during pregnancy is more serious, and most of the patients have increased heart rate, dyspnea and decreased blood pressure when there is massive hemorrhage of upper digestive tract. Paying more attention to the changes of heart rate, blood pressure, blood oxygen and respiration can predict the occurrence of upper GI bleeding and improve the success rate of treatment [3]. Patient 2: Vital signs on admission, HR102 - 122 bpm, RR18 - 26 bpm, SPO2 $90 \%-98 \%$, with two venous accesses opening. And active symptomatic care was given before the symptoms of hematemesis occurred to prevent the condition from worsening such as shock and DIC. Patient 1: Vital signs on admission, BP136/92 mmHg. ECG monitoring showed that blood pressure fluctuated between 138 - 152/70 - $95 \mathrm{mmHg}$. Nifedipine sustained-release tablets were given orally, with blood pressure reduced to 120 - 139/68 - $88 \mathrm{mmHg}$. Hypertensive disorder is a common complication in patients with pregnancy complicated with liver cirrhosis, with an incidence of $18 \%-81.8 \%$ [2] [4]. Patient 1 with high blood pressure, was given timely medication, control of dosage, and informed of low-salt and low-fat diet. The blood pressure was maintained well, and there was 
no acute upper GI bleeding.

2) Life care: Pregnant women in the first and second trimester prefer spicy food because of morning sickness, but patients with upper GI bleeding complicated with liver cirrhosis should keep a bland diet instead of anything raw or cold, salty and spicy, to reduce the stimulation of digestive tract veins, keep the stool unobstructed to avoid the increase of abdominal pressure caused by constipation. Patients with severe varicose veins should avoid intense coughing or expectoration, reducing the risk of gastrointestinal bleeding. Patient 2: the appetite was improved after symptomatic treatment, and the amount of upper GI bleeding reached $600 \mathrm{~mL}$ on the third day after hospitalization. Upper gastrointestinal variceal ligation was performed on the same day, and the curative effect was significant.

3) Perioperative nursing of upper digestive tract vein ligation. a) preoperative preparation: Blood routine, blood coagulation routine, blood type examination and electrocardiogram before operation was performed. Fasting 6 hours before operation was implemented. Nurses can explain the principle and specific process of ligation to patients and their families to reduce their preoperative tension, anxiety and fear, and allay doubts and worries. b) postoperative nursing: Patients are informed that incorrect diet is an important cause of esophageal variceal bleeding and rebleeding in patients with liver cirrhosis [5]. Patients are mainly instructed to stay in bed and raise the head of the bed. Studies have shown that lying in bed and raising the head of the bed about 30 after ligation can effectively prevent gastric acid reflux from stimulating the esophagus [6]. Fast within 24 hours after operation. A small amount of warm and cold boiled water was given 24 - 28 hours after operation, and a small amount of warm and cold liquid diet was given to patients 3 - 6 days after operation, such as rice noodles, porridge, etc., and gradually transferred to soft food when the condition improved.

\subsection{Psychological Nursing}

The two patients with chronic diseases, having a low probability of successful pregnancy, had a high expectation for the success of this pregnancy. But in the decompensated period of liver cirrhosis, due to metabolic disorders caused by liver cirrhosis, the incidence of abortion, stillbirth, premature delivery, hypertensive disorder complicating pregnancy, postpartum hemorrhage and maternal mortality increased during pregnancy [7]. Patient 1 is a 24-year-old young patient who is faced with the difficult choice of giving up the fetus in the first pregnancy. Intrauterine death had caused feelings of sadness, depression and even despair among the patient 2 and her family. Nursing staff should understand the psychological state of patients and their families, inquire about the current demands and their understanding of the disease, answer patients' questions patiently and meticulously, and pay attention to keeping the ward and bed clean and tidy environment. improve the inner comfort and intimacy of patients [8]. 


\subsection{Preparation before Clearing the Palace}

In the decompensated stage of liver cirrhosis, due to the significant decrease of liver function, induced labor in the first and second trimester should implement by physical methods instead of oral drug. Patient 2: the condition worsened and upper GI bleeding increased after hospitalization. In order to prevent the further deterioration and reduce the damage of liver function caused by drugs, endoscopic esophageal variceal ligation and uterine curettage were performed under endotracheal general anesthesia on the 3rd day after admission. Forbid drinking for 6 hours before operation, increase the amount of $500 \mathrm{ml}$ rehydration to fill the bladder and ensure that the uterus can be fully exposed.

\subsection{Nursing in the Process of Induced Labor}

Patient 1: Due to the good cervical condition and the signs of threatened labor, the patient was evaluated to be able to deliver in a short time, and the foley balloon was placed to induce labor. Foley balloon is a mechanical device for promoting cervical maturation, which produces stable and mild tension on the cervix, which can stimulate the secretion of endogenous prostaglandins and induce uterine contraction [9].

Before placing the foley balloon, the purpose, process and related cooperation measures of the balloon placement should be explained to the patient in detail. During the exposed part of the balloon, the nursing staff should ensure that the catheter is pulled to the outside of the cervix mouth of the patient and placed correctly, which can effectively reduce the clinical pain of the patient.

Double-tube venous channels were established after balloon placement, and patients were closely observed for discomfort, vaginal bleeding, uterine contraction, natural water breaking and so on. When balloon prolapse was found, the expulsion of embryonic tissue was checked in time. After the expulsion of the fetus and its accessories, check the uterine contraction immediately, check whether the fetal placenta is complete, and use oxytocin to promote uterine contraction in time to prevent postpartum hemorrhage. When the placenta is found to be incomplete, nurses should assist the doctor to clear the palace in time to reduce the occurrence of bleeding. In the process of induced labor, nurses should pay attention to patients' feelings, and give psychological support such as humanistic care and guidance of non-drug analgesia. Patient 1 was given intramuscular injection of carboprost aminobutyriol $250 \mu \mathrm{g}$ while bleeding 350 $\mathrm{mL}$ in 24 hours during births.

\subsection{Routine Nursing after Induced Labor}

After induced labor, the vital signs, vaginal bleeding and uterine contraction were closely observed and recorded in detail, and the amount of 24-hour postpartum bleeding was counted. The placenta can produce placental prolactin at the 16th week. Pay attention to the production to see if the breast has increased milk, so as to avoid drug demilking to increase the metabolic burden of the liver. 
External application drugs such as borneol mirabilite milk can be mixed to make $100 \mathrm{~g}$ packets, which can be applied twice a day to the swollen breast until the swelling goes down.

\subsection{Monitoring and Nursing of Complications}

1) Prevention and nursing of postpartum hemorrhage: Due to the decrease of liver function and the synthesis of coagulation factors in the decompensated stage of liver cirrhosis, postpartum hemorrhage in the decompensated stage of liver cirrhosis is the main complication. Shortening the stage of labor, intraoperative administration of uterine contraction, placement of uterine cavity packing balloon can reduce postpartum hemorrhage. After operation, hemostatic drugs, coagulation factors and continuous infusion of oxytocin were given. vaginal bleeding was closely observed; Maternal complaints were paid attention to, and uterine bleeding was monitored by color Doppler ultrasound in time. Hemoglobin decreased significantly in 2 cases after induced labor, and red blood cells and fresh frozen plasma were infused, which was related to mild anemia and serious disturbance of blood coagulation before delivery. But there were no serious complications such as postpartum hemorrhage, shock, DIC and so on.

2) Prevention and nursing of hepatic encephalopathy and hepatorenal syndrome: Hepatic encephalopathy and hepatorenal syndrome can be induced by bleeding, water/electrolyte imbalance, or surgical trauma caused by bronchoscopy during labor induction [10]. Postpartum liver sex encephalopathy prodromal period shows spirit abnormal excitement, gibberish, abnormal behavior. Hepatorenal syndrome is characterized by hypourocrinia or anuria, with concentrated appearance and rapid changes in systemic edema. In addition to monitoring the vital signs, patients should be tested for consciousness and behavior, and be asked questions of "positioning", "timing" and "orientation" every day. If there is no answer to the question, or a precursor of liver disease and encephalopathy, nurses should report to the doctor timely, and monitor the urine volume, blood electrolyte, blood creatinine and blood urea nitrogen of the patient [11].

3) Anti-infection care: In the decompensated stage of cirrhosis, according to decrease of protein synthesis, low resistance, and increased probability of puerperal infection., nurses should attach great importance to preventing infection. 2 cases were delivered through vagina, and perineal washing was performed with disinfectant during lochia. In the use of multiple antibiotics, attention should be paid to drug compatibility contraindications and accurate time of administration to ensure reasonable distribution of drug concentration of antibiotics. Instruct patients and their family members to enhance health awareness, wash hands and bathe frequently to reduce the way of infection transmission. Abdominal puncture and drainage of ascites were performed on the 3rd day after delivery in case 1. Nursing care of the drainage tube and publicity and health education were strengthened. The tube was successfully extubated before discharge, and no re- 
lated infection occurred.

\subsection{Discharge Guidance}

Patients with decompensated cirrhosis should pay attention to rest, high protein and calorie intaking. Daily perineal cleansing, no bath, sexual life for 6 weeks; Patients should choose physical contraception to avoid contraceptive drugs aggravate liver damage; Pay attention to lochia; Keep a good attitude and a stable mood; 2 weeks later, the obstetrics clinic was revisited. Transfer to hepatology department.

\section{Conclusion}

Upper gastrointestinal bleeding in decompensated liver cirrhosis is the most serious complication of liver cirrhosis. Without early active treatment, the annual mortality is up to $46.7 \%$ [12]. When it occurred in the first and second trimester, hemostatic treatment and labor induction should be carried out to terminate pregnancy in the appropriate time. Nursing care of gastrointestinal hemorrhage, life guidance and psychological nursing should be strengthened before induced labor. Postpartum hemorrhage and liver cirrhosis-related complications should be prevented and treated to improve liver and blood coagulation function and improve the prognosis of patients.

\section{Conflicts of Interest}

The authors declare no conflicts of interest regarding the publication of this paper.

\section{References}

[1] Tolunay, H.E., Aydın, M., Cim, N., Boza, B., Dulger, A.C., Yıldızhan, R. and Caronna, R. (2020) Maternal and Fetal Outcomes of Pregnant Women with Hepatic Cirrhosis. Gastroenterology Research and Practice, 2020, Article ID 5819819. https://doi.org/10.1155/2020/5819819

[2] Jena, P., Sheela, C.N., Venkatachala, R.P. and Devarbhavi, H. (2017) Obstetric Outcome in Women with Chronic Liver Disease. The Journal of Obstetrics and Gynecology of India, 67, 263-269. https://doi.org/10.1007/s13224-016-0959-y

[3] Mansour, D. and McPherson, S. (2018) Management of Decompensated Cirrhosis. Clinical Medicine Journal, 18, s60-s65. https://doi.org/10.7861/clinmedicine.18-2-s60

[4] Pergolini, M.S. (2009) The Management of Hypertensive Crises: A Clinical Review. Clinical Therapeutics, 160, 151-157.

[5] Guo, Y.L., Xu, B.H., Liu, X., et al. (2018) Risk Factors Analysis of Early Rebleeding after Endoscopic Treatment of Esophageal Varices. Chinese Journal of Digestive Endoscopy, 35, 89-93.

[6] Karvellas, C.J., Bagshaw, S.M., Karvellas, C.J., et al. (2014) Advances in Management and Prognostication in Critically Ill Cirrhotic Patients. Current Opinion in Critical Care, 20, 210-217. https://doi.org/10.1097/MCC.0000000000000067

[7] Mikolasevic, I., Filipec-Kanizaj, T., Jakopcic, I., et al. (2018) Liver Disease during 
Pregnancy: A Challenging Clinical Issue. Medical Science Monitor, 24, 4080-4090. https://doi.org/10.12659/MSM.907723

[8] Woodland, H., Hudson, B., et al. (2019) Palliative Care in Liver Disease: What Does Good Look Like? Frontline Gastroenterology, 11, 218-227.

https://doi.org/10.1136/flgastro-2019-101180

[9] Adhikari, E.H., Nelson, D.B., McIntire, D.D. and Leveno, K.J. (2020) Foley Bulb Added to an Oral Misoprostol Induction Protocol: A Cluster Randomized Trial. Obstetrics \& Gynecology, 136, 953-961.

https://doi.org/10.1097/AOG.0000000000004123

[10] Peng, I.T., Chung, M.T. and Lin, C.C. (2018) Severe Postpartum Hemorrhage Complicated with Liver Infarction Resulting in Hepatic Failure Necessitating Liver Transplantation. Case Reports in Obstetrics and Gynecology, 2018, Article ID 2794374. https://doi.org/10.1155/2018/2794374

[11] Patidar, K.R. and Bajaj, J.S. (2015) Covert and Overt Hepatic Encephalopathy: Diagnosis and Management. Clinical Gastroenterology and Hepatology, 13, 2048-2061. https://doi.org/10.1016/j.cgh.2015.06.039

[12] Zhou, Y.J., Zheng, J.N., Zhou, Y.F., et al. (2017) Development of a Prognostic Nomogram for Cirrhotic Patients with Upper Gastrointestinal Bleeding. European Journal of Gastroenterology \& Hepatology, 29, 1166-1173.

https://doi.org/10.1097/MEG.0000000000000943 